\title{
Cambridge Low Frequency Surveys
}

\author{
D.A. Green \\ Mullard Radio Astronomy Observatory, Cavendish Laboratory, \\ Madingley Road, Cambridge CB3 OHE, U.K.
}

\begin{abstract}
Over many years low-frequency radio surveys have been made in Cambridge that cover much of the northern sky at both 38 and $151 \mathrm{MHz}$. The characteristics of the earlier surveys - 6C (at $151 \mathrm{MHz}, \delta$ above $+30^{\circ}$ ) and $8 \mathrm{C}\left(\right.$ at $38 \mathrm{MHz}, \delta$ above $+60^{\circ}$ ), both with a resolution of about 4 arcmin - are reviewed, together with the results from more recent surveys made at $151 \mathrm{MHz}$, with a resolution of about 1.2 arcmin with the Cambridge Low-Frequency Synthesis Telescope (CLFST). These surveys include the 7C survey, which includes low declinations to complement $6 \mathrm{C}$, selected regions at higher declinations, variability studies, and the $7 \mathrm{C}(\mathrm{G})$ survey of much of the northern Galactic plane.
\end{abstract}

\section{Introduction}

Here I review several low-frequency radio surveys observed at the Mullard Radio Astronomy Observatory, Cambridge, UK since the 1970s. These surveys are the 6C and 8C surveys, both made with non-tracking telescopes with a resolution of $\sim 4 \operatorname{arcmin}$ at 151 and $38 \mathrm{MHz}$ respectively, and the 7C surveys made with the Cambridge Low-Frequency Synthesis Telescope (CLFST) at $151 \mathrm{MHz}$, with a resolution of $\sim 1.2$ arcmin. Some of the other observations made with the CLFST are also briefly discussed. Further information about these surveys, including details of how to obtain source lists, is available on the Web, via: "http://www.mrao.cam.ac.uk/surveys/".

\section{The $6 \mathrm{C}$ survey}

The 6C survey, made at $151 \mathrm{MHz}$, was observed in $1975-78$, to provide a 4arcmin resolution survey of most the northern sky above declinations of $\sim 30^{\circ}$. The telescope used to make this survey was an east-west, aperture synthesis telescope, which was rather unusual in that the antennas did not track. Rather than tracking fixed points on the sky for 12-hour syntheses, as is conventional with east-west aperture synthesis telescopes, observations were made by observing at fixed declinations and hour angles. This observing procedure results in a significantly simpler, and cheaper telescope, but requires more complicated analysis to combine data taken from different pointings into the syntheses of different fields on the sky.

The parameters of the $6 \mathrm{C}$ survey are given in Table 1 . The $6 \mathrm{C}$ survey was published from 1985 - see Baldwin et al. (1985) for the first portion of 
Table 1. The parameters of the $6 \mathrm{C}$ survey.

\begin{aligned} & \hline frequency: $151.5 \mathrm{MHz} \\ &$ observed: $1975-78 \\ &$ number of antennas: 50 (quad yagis) \\ & number of baselines: 446 \\ & mounting: alt-az (no drive) \\ & maximum baseline: $1.4 \mathrm{~km}$ east-west $(\equiv 692 \lambda) \\ &$ resolution: $4.2 \times 4.2 \operatorname{cosec}(\delta) \operatorname{arcmin}^{2} \\ &$ field of view: $\sim 17^{\circ} \\ & 1-\sigma$ sensitivity: $2^{20} \mathrm{mJy} \\ &$ number of sources: 35,000 \\ & published: $1985-1993 \\ &$\hline \hline\end{aligned}

the survey, which describes the telescope, its operation and the data analysis in detail, and Hales, Baldwin, \& Warner (1988), Hales et al. (1990, 1991, 1993) and Hales, Baldwin, \& Warner (1993) for the subsequent portions of the survey. In total the published survey consists of images of several hundred 'panels', each $\sim 3 \times 6 \mathrm{deg}^{2}$, together with associated source lists giving details of thirty five thousand compact sources.

The 6C telescope was also used for a search for red-shifted $\mathrm{HI}$ at $151 \mathrm{MHz}$ by Bebbington (1986), who obtained a limit of around $10^{15} M_{\odot}$ for primordial pancakes at this redshift.

\section{The $8 \mathrm{C}$ survey}

The $8 \mathrm{C}$ survey, made at $38 \mathrm{MHz}$, was observed in 1986--87, i.e. near solar minimum, to provide a 4 -arcmin resolution survey complementary to $6 \mathrm{C}$ (for declinations above $\sim 60^{\circ}$ ). (Note: the telescope used for the $8 \mathrm{C}$ survey utilised much of the electronics on the CLFST telescope, see the next section, which was constructed in the early 1980s.) This survey was generally similar in operation to $6 \mathrm{C}$, having non-tracking antennas observing fixed declinations and hour angles (one at the north celestial pole, and three at declination of $72^{\circ}$ ). One difference between the $8 \mathrm{C}$ and $6 \mathrm{C}$ surveys was that self-calibration was needed to remove ionospheric phase variations in the lower frequency $8 \mathrm{C}$ survey.

The parameters of the $8 \mathrm{C}$ survey are given in Table 2 . The $8 \mathrm{C}$ survey was published by Rees (1990a), who presented images, for over seventy 'panels' at $38 \mathrm{MHz}$, and source lists and by Hales et al. (1995) who presented a consolidated, and corrected source list.

An interesting consequence of the calibration of the $8 \mathrm{C}$ survey was - Rees (1990b) - that it revealed difficulties in reconciling the observations with the flux density scale of Baars et al. (1977). The results imply that the frequencydependent fading of Cas A as used by Baars et al. is not correct, and uncertainties in our knowledge of this behaviour of Cas A limit the accuracy of flux density scales based on Cas A at both low frequencies, and high frequencies. 
Table 2. Parameters of the $8 \mathrm{C}$ survey

\begin{aligned} & \hline frequency: $38 \mathrm{MHz} \\ &$ observed: $1986-87 \\ &$ number of antennas: 58 (yagis) \\ & number of baselines: 742 \\ & mounting: alt-az (no drive) \\ & maximum baseline: $4.6 \mathrm{~km}$ nearly east-west $(\equiv 572 \lambda) \\ &$ resolution: $4.5 \times 4.5 \operatorname{cosec}(\delta) \operatorname{arcmin}^{2} \\ &$ field of view: $\sim 30^{\circ} \\ & 1-\sigma$ sensitivity: $\gtrsim 200 \mathrm{mJy} \\ &$ number of sources: 5,000 \\ & published: 1990 and 1995 \\ & \hline \hline\end{aligned}

\section{The CLFST and the $7 \mathrm{C}$ surveys}

After the completion of the $6 \mathrm{C}$ observations, the yagi antennas of the $6 \mathrm{C}$ telescope were, in the early 1980 s, incorporated into a new, higher resolution 151$\mathrm{MHz}$ telescope, which was called the Cambridge Low-Frequency Synthesis Telescope (CLFST). Unlike the 6C and 8C telescopes, this is a conventional telescope with 60 tracking antennas. The antennas are in groups controlled by local microcomputers in 'huts' - actually portable toilet buildings! - distributed over a $\sim 4.6 \mathrm{~km}$, approximately east-west baseline, to give an increased resolution of $1.2 \times 1.2 \operatorname{cosec}(\delta) \operatorname{arcmin}^{2}$.

The characteristics of the CLFST mean that the analysis of its observations has some peculiarities: i) the wide field-of-view $\left(\sim 17^{\circ}\right)$ means that bright sources a long way away from the field centre need to removed; ii) self-calibration is needed to remove ionospheric phase variations; and iii) the fact that the telescope is not exactly east-west means that the synthesised beam varies across images, for which a customised 'beamset' technique (see Waldram \& McGilchrist 1990; Waldram \& Riley 1993) has been developed to determine the parameters of compact sources.

The CLFST has been used for a variety of observations, most notably various 7C surveys (e.g. Riley, Waldram, \& Riley 1999 and references therein), which include: i) the Low-Declination 7C survey - to extend $6 \mathrm{C}$ down to $\delta \sim 20^{\circ}$ (Waldram et al. 1996); ii) repeated observations of several fields for variability studies (e.g. McGilchrist \& Riley 1990; Riley \& Warner 1994; Minns \& Riley 2000); iii) the $7 \mathrm{C}(\mathrm{G})$ survey of most of the northern Galactic plane (see Vessey \& Green 1998); iv) surveys of selected fields (e.g. Abell clusters; ROSAT deep fields, etc). The variability studies reveal flux density variations of typically $15-25$ per cent on timescales of 1 to 12 years, with the fractional variability and variability timescales varying with Galactic latitude (Minns \& Riley 2000), consistent with refractive interstellar scintillation.

In addition to the survey and variability observations, the CLFST has also been used for a variety of unconventional observations. These include studies of the bulk expansion of the SNR Cas A, from contraction of well-defined fea- 
tures observed in the visibility plane (Agüeros \& Green 1999), and searches for 'prompt' radio emission from GRBs (e.g. Dessenne et al. 1996). For the latter observations, a special observing mode for the CLFST was developed, where the antennas were divided into seven groups, which each observed a different position on the sky. In this way it was possible to obtain limits on any prompt radio emission from GRBs, including some real-time limits.

Acknowledgments. The various telescopes and surveys described above are the result of the expertise and effort expended over many years by very many Cambridge radio astronomy personnel, whom it is not possible to acknowledge individually.

\section{References}

Agüeros, M.A. \& Green, D.A. 1999, MNRAS, 305, 957

Baars, J.W.M. Genzel, R. Pauliny-Toth, I.I.K. \& Witzel, A. 1977, A\&A, 61, 99

Baldwin, J.E. Boysen, R.C. Hales, S.E.G. Jennings, J.E. Waggett, P.C. Warner, P.J. \& Wilson, D.M.A. 1985, MNRAS, 217, 717

Bebbington, D.H.O. 1986, MNRAS, 218, 577

Dessenne, C.A.-C., Green, D.A. Warner, P.J. Titterington, D.J. Waldram, E.M. Barthelmy, S.D. Butterworth, P.S. Cline, T.L. Gehrels, N. Palmer, D.M. Fishman, G.J. Kouveliotou, C. \& Meegan, C.A. 1996, MNRAS, 281, 977

Hales, S.E.G. Baldwin, J.E. \& Warner, P.J. 1988, MNRAS, 234, 919

Hales, S.E.G. Masson, C.R. Warner, P.J. \& Baldwin, J.E. 1990, MNRAS, 246, 256

Hales, S.E.G. Mayer, C.J. Warner, P.J. \& Baldwin, J.E. 1991, MNRAS, 251, 46

Hales, S.E.G. Baldwin, J.E. \& Warner, P.J. 1993, MNRAS, 263, 25

Hales, S.E.G. Masson, C.R. Warner, P.J. \& Baldwin, J.E. Green, D.A. 1993, MNRAS, 262, 1057

Hales, S.E.G. Waldram, E.M. Rees, N. \& Warner, P.J. 1995, MNRAS, 274, 447

McGilchrist, M.M. \& Riley, J.M. 1990, MNRAS, 246, 123

Minns, A.R. \& Riley, J.M., 2000, MNRAS, in press

Rees, N. 1990a, MNRAS, 244, 233

Rees, N. 1990b, MNRAS, 243, 637

Riley, J.M.W. Waldram, E.M. \& Riley, J.M. 1999, MNRAS, 306, 31

Riley, J.M. \& Warner, P.J. 1994, MNRAS, 269, 166

Vessey, S.J. \& Green, D.A. 1998, MNRAS, 294, 607

Waldram, E.M. \& McGilchrist, M.M. 1990, MNRAS, 245, 532

Waldram, E.M. \& Riley, J.M. 1993, MNRAS, 265, 853

Waldram, E.M. Yates, J.A. Riley, J.M. \& Warner, P.J. 1996, MNRAS, 282, 779 